\title{
Adaptación en el paciente con amputación: una mirada desde la teoría de Roy
}

\author{
Adaptation in the patient with amputation, a look from Roy's theory
}

\author{
Margarita Lazcano-Ortiz, ${ }^{a}$ Sinahi Pelcastre-Portillo ${ }^{b}$, Xóchitl Martínez-Hernández ${ }^{c}$, Brenda \\ L. Lazcano-González ${ }^{d}$, Blanca E. Vargas-Castro ${ }^{e}$, Erika Fragoso-Gómez ${ }^{f}$,Reyna C. Jiménez- \\ Sánchez ${ }^{g}$.
}

\begin{abstract}
:
Objective: To determine the level of coping and adaptation of patients with amputation. Material and method: Descriptive crosssectional quantitative study. Sampling not probabilistic for convenience. The constant sample of 15 people with different types of amputation, originating in the state of Hidalgo. The Callista Roy Coping and Adaptation Process Measurement Scale instrument was used, with a cronbach alpha reliability coefficient of .746. Informed consent was provided. For data analysis, use the SPSS version 21 program. Results: The average age was 47 years $(\mathrm{SD}=16.2) ; 25-78$. With total participation of the male gender $(100 \%)$. It is observed that the recursive and centered factor is high (100\%); in the physical and focused factor $60 \%$ shows low capacity for coping and adaptation, in the alert process factor there is a high capacity for adaptation $87 \%$; in systematic processing $60 \%$ adaptive capacity and coping; in the factor knowing and relating $73 \%$ with high capacity for coping and adaptation. As a general result, people have a high capacity for coping and adaptation with 93\%. Conclusions: Most patients face the problem from the beginning and analyze all possible options to solve the problem; However, patients with low levels of adaptation and coping have difficulty distinguishing the root of the problem, they see the problem as complex and they have difficulty applying strategies that have served them in past experiences.
\end{abstract}

Keywords:

Adaptation, Amputation, Callista Roy, Coping

\section{Resumen:}

Objetivo: Determinar el nivel de afrontamiento y adaptación de pacientes con amputación. Material y método: Estudio descriptivo, transversal, cuantitativo. Muestreo no probabilístico por conveniencia. La muestra consta de 15 personas con diferentes tipos de amputación, originarios del estado de Hidalgo. Se utilizó el instrumento Escala de medición del Proceso de Afrontamiento y Adaptación de Callista Roy, con un coeficiente de confiabilidad alfa de cronbach de .746. Se proporcionó consentimiento informado. Para el análisis de datos se usó el programa SPSS versión 21. Resultados: El promedio de edad fue de 47 años $\quad(\mathrm{DE}=16.2)$; 2578. Con participación total del género masculino (100\%). Se observa que en el factor recursivo y centrado es alto (100\%); en el factor físico y enfocado el $60 \%$ muestra baja capacidad de afrontamiento y adaptación, en el factor proceso de alerta se presenta alta capacidad 87\%; en procesamiento sistemático baja capacidad de afrontamiento y adaptación 60\%; en el factor conociendo y relacionando $73 \%$ con alta capacidad de afrontamiento y adaptación. Como resultado general las personas mostraron alta capacidad de afrontamiento y adaptación con un 93\%.Conclusiones: La mayoría de los pacientes hacen frente al problema desde un principio y analizan todas las posibles opciones para solucionar el problema; sin embargo, los pacientes con bajo nivel de afrontamiento y

\footnotetext{
Autor de Correspondencia, Universidad Autónoma del Estado de Hidalgo, https://orcid.org/0000-0002-9653-9291. Email: margarita_lazcano@uaeh.edu.mx

b Universidad Autónoma del Estado de Hidalgo, https://orcid.org/0000-0002-0364-7104. Email: sitni97@ outlook.com. c Universidad Autónoma del Estado de Hidalgo, https://orcid.org/0000-0002-3463-0348. Email: ma358060@uaeh.edu.mx d Universidad Autónoma del Estado de Hidalgo, https://orcid.org/0000-0003-4100-1176. Email: la355957@uaeh.edu.mx e Universidad Autónoma del Estado de Hidalgo, https://orcid.org/0000-0002-8629-3443. Email: va250271@uaeh.edu.mx ${ }^{\text {f } U n i v e r s i d a d ~ A u t o ́ n o m a ~ d e l ~ E s t a d o ~ d e ~ H i d a l g o, ~ h t t p s: / / o r c i d . o r g / 0000-0002-0409-9939 . ~ E m a i l: ~ e m a i l: c r i s t i j i @ ~ h o t m a i l . c o m ~}$ g Universidad Autónoma del Estado de Hidalgo, https://orcid.org/0000-0001-9264-8514. Email: fr326605@uaeh.edu.mx
} 
adaptación presentan dificultad para distinguir cual es la raíz del problema, ven el problema muy complejo y se les obstaculiza aplicar estrategias que les han servido en experiencias pasadas.

\section{Palabras Clave:}

Adaptación, Amputación, Callista Roy, Afrontamiento

\section{Introducción}

Un problema en nuestro "país" son las amputaciones que conllevan a discapacidad y mortalidad prematura. En México por parte del INEGI [1] en 2014 la prevalencia de la discapacidad fue de $6 \%$, los principales detonantes de discapacidad son las enfermedades (41.3\%) y la edad avanzada (33.1\%). También reporta en el 2014 que existían 785000 personas amputadas de una o varias extremidades. Diversas fuentes indican que el número total de amputados de diferentes partes del cuerpo en el 2014 fue de alrededor de 935000 [2].

El pie diabético es una de las complicaciones más comunes asociadas con la diabetes mellitus (DM), se estima que aproximadamente del 15 al $25 \%$ de los pacientes con DM desarrollan pie diabético durante el curso de su enfermedad [3,4]. Sin embargo, esta condición de discapacidad es una de las causas más frecuentes de hospitalización para las personas con DM.

En la actualidad el número de pacientes amputados en el mundo occidental mantiene un incremento anual debido principalmente al envejecimiento de la población, que presenta con la edad algunos problemas crónicos, como diabetes y enfermedad vascular periférica. Además, se observa que el mayor número de personas con pérdida de extremidades (90\% corresponde al miembro inferior) se encuentra entre los 60 y 75 años de edad. [2]

Perder alguna extremidad implica generar cambios extremos en el estilo de vida y desarrollar un proceso de afrontamiento y adaptación que le permitan a la persona conducirse en poco tiempo a vivir con una discapacidad. Es importante fomentar un ambiente en el que se brinde apoyo psicológico, emocional, social, e intervenir de forma integral en este grupo de personas para lograr recuperar las funciones perdidas tomando en cuenta los aspectos tanto físicos como sociales. [5]

La presente investigación se guía con la Teoría del proceso de adaptación por el que afrontan las personas que han sufrido un evento estresante [5]

\section{Marco teórico}

\section{Modelo de Adaptación de Roy}

El modelo de adaptación de Roy muestra como el ser humano posee las capacidades y recursos necesarios para enfrentar su proceso de salud-enfermedad y así lograr un nivel de adaptación a ciertas situaciones.

La adaptación para Callista Roy la define como "el proceso y el resultado por medio de la cual las personas con pensamientos y sentimientos en forma individual o grupal, utilizan la conciencia consciente y eligen para crear una integración humana y ambiental" [5]

El modelo señala que el ser humano es altamente capaz de adaptarse a cualquier ambiente y crear cambios en "él". La capacidad de responder positivamente a estos cambios es una función del nivel de adaptación del sistema humano [5.6.7]

En realidad, para la valoración del usuario es necesario verlo como la conformación de un todo, de modo fisiológico y psicosocial, desde luego que el interés no sea solo por su estado de salud, sino también de lo que piensa el paciente, lo que siente frente a la enfermedad y las expectativas que tiene del cuidado de enfermería, conocer estos puntos son fundamentales para establecer estrategias y de esta manera promover la adaptación del individuo, el cual es el principal objetivo del modelo de adaptación. [6]

\section{Metaparadigma del Modelo de Roy}

El modelo de Roy plantea cuatro conceptos en el metaparadigma que permiten interactuar entre si para lograr la adaptación [6].

Ser humano: constitución de las partes centrada en un todo para conseguir un propósito específico, trabajando en función de la interdependencia de cada una de las partes.[5,6] Desde luego, las personas se adaptan a diversos procesos de manera diferente y esto dependerá 
siempre de las experiencias adquiridas a lo largo de la vida, por esta razón el personal de enfermería debe considerar al ser humano como un ser único, autónomo, digno, libre y especial por el hecho de formar parte de un contexto diferente del resto de la sociedad y de la cual no se puede separar $[7,8,9]$

Medio ambiente: Son todas las condiciones, circunstancias e influencias que rodean y afectan el desarrollo y el comportamiento de los seres humanos como sistemas adaptativos con particular consideración de la persona y de los recursos del mundo.[7,8,9] Bueno, desde esta afirmación el ambiente del ser humano, debe ser tratado como un ser holístico, no solo se limita al ambiente externo, es decir las personas, lugares, actividades que realiza cotidianamente, sino que también al ambiente interno, como lo son sus experiencias, sus valores, su forma de ver la vida, etc. $[5,6]$

Salud: proceso de ser y llegar a ser un todo integrado.

Enfermería: sistema de conocimientos teóricos cuya función principal es facilitar el proceso de adaptación, sirviendo de guía para el individuo [6].

\section{Estímulos del modelo de Roy}

Por tanto, el modelo de adaptación de Roy hace referencia a tres estímulos presentes en el ambiente los cuales son: focales, contextuales y residuales. [5,6]

Estimulo focal: es el nivel de estímulo respuesta inmediata que consiste en un objeto o situación que se presenta en la conciencia de la persona, la cual enfoca toda su energía tratando de enfrentarlo.

Estimulo contextual: es el nivel de estímulo que se encuentra en el ambiente y el cual puede favorecer 0 empeorar la situación del usuario para poder adaptarse a la situación.

Estimulo residual: estimulo producto de experiencias pasadas, creencias y expectativas de vida, pues abarca el ambiente tanto interno como externo, y cuyo efecto no son claras en una situación presente $[5,6]$

\section{Afrontamiento}

Es importante señalar que el personal de enfermería necesita identificar los conceptos de afrontamiento y adaptación para la calidad de vida de un individuo, porque son procesos dinámicos en los cuales se realiza la integración entre la persona y el medio ambiente donde se generan estilos y estrategias para afrontar una situación $[10,11]$

Además, el afrontamiento es entendido como los esfuerzos comportamentales y cognitivos que realiza la persona para atender las demandas del ambiente, actúan como un todo para mantener sus procesos vitales y su integridad. [10,11]

Roy desarrolló una teoría sobre el "Proceso de afrontamiento y adaptación" basada en su modelo conceptual, en el cual describe las estrategias que utiliza la persona para responder a las influencias de los cambios ambientales [5]

Además, sustenta la teoría en una serie de premisas: a) La persona es un ser bio-psicosocial, por lo tanto, la persona está en constante interacción con el entorno cambiante.

b) Al afrontar un mundo cambiante la persona usa mecanismos innatos y adquiridos, cuyo origen es biológico, psicológico y social;

c) La salud y la enfermedad son dimensiones inevitables en la vida de las personas $y$;

d) Para responder positivamente a los cambios del entorno la persona debe adaptarse [10].

Las estrategias comportamentales buscan controlar el estímulo de manera directa, actuando frente al problema que la persona considera que es susceptible de modificar, siendo estas evasivas cuando la persona siente que no puede actuar ante el estímulo y lo rechaza de forma consciente o inconsciente [5].

Para Callista Roy la salud es el proceso de ser y llegar a ser un todo integrado, es decir, la salud es sinónimo de adaptación. El proceso de adaptación de cada persona será diferente dependiendo de las circunstancias que existan en su ambiente, esto conlleva a que los seres humanos cada vez sean más complejos y tengan mayor crecimiento. [6,7]

\section{Objetivo General}

Determinar el nivel de afrontamiento y adaptación de pacientes con amputación

\section{Objetivo Especifico}

Identificar el estilo de afrontamiento y adaptación que presenta el paciente con amputación

Evaluar la capacidad de afrontamiento y adaptación de pacientes que presentan amputación 


\section{Material y Método}

Se realizó un estudio no experimental, transversal, cuantitativo, descriptivo. La muestra estuvo conformada por 15 personas. El tipo de muestreo fue no probabilístico por conveniencia. Se invitó a participar en el estudio dando a conocer los objetivos y que cumplieran con criterios de inclusión tales como: originarios de Hidalgo, ambos sexos, cualquier nivel de escolaridad, que presentaran amputación de algún miembro corporal. Se entregó consentimiento informado.

El procesamiento y análisis de datos se realizó a través del paquete estadístico SPSS versión 21.

\section{Instrumento}

Se aplicaron dos instrumentos: un cuestionario para datos sociodemográficos y la escala de medición Proceso de Afrontamiento y Adaptación de Callista Roy. [Coping Adaptación Processing Scale (CAPS)], consta de 47 ítems, con 4 opciones de respuesta desde 1 = Nunca; hasta 4 = Siempre. Con cinco factores de afrontamiento, los tres primeros miden los comportamientos, las reacciones y los recursos de afrontamiento que utilizan las personas y los dos últimos miden estrategias empleadas para sobrellevar la situación: Factor 1; recursivo y centrado, Factor 2; físico y enfocado, Factor 3; proceso de alerta, Factor 4; procesamiento sistémico de la información, Factor 5; conociendo y relacionando. [12]

Con un Coeficiente de Confiabilidad Alpha de Cronbach de 0.746 .

\section{Resultados}

El promedio de edad fue de $47.6(\mathrm{DE}=16.295 ; 25-78) \mathrm{con}$ predominio en el sexo masculino (100\%). Se observa en la tabla 1 los datos sociodemográficos, donde menos de la mitad $(40 \%)$ de los participantes están casados, seguido de una tercera parte (33.3\%) que se encuentran en unión libre, en relación al nivel de escolaridad se observa que más de la mitad (53.3\%) sólo terminaron la primaria y una mínima parte $(6.7 \%)$ tiene estudios universitarios. Con respecto a la ocupación el $33.3 \%$ son trabajadores asalariados mientras que poco menos de la cuarta parte (20\%) son pensionados y menos de la mitad $(46.7 \%)$

son autoempleados. El $80 \%$ profesa la religión católica, el $6.7 \%$ profesa religión cristiana, igual que la religión mormona y testigos de Jehová el $6.7 \%$.
Tabla 1 Datos Sociodemográficos

\begin{tabular}{ccc}
\hline & $f r$ & $\%$ \\
\hline Estado civil & 6 & 40 \\
Casados & 5 & 33.3 \\
Unión libre & 3 & 20.0 \\
Solteros & 1 & 6.7 \\
Viudo & & \\
Escolaridad & 8 & 53.3 \\
Primaria & 4 & 26.7 \\
Secundaria & 2 & 13.3 \\
Preparatoria & 1 & 6.7 \\
Universidad & & \\
Ocupación & 5 & 33.3 \\
Trabajador & & \\
asalariado & 3 & 20 \\
Pensionado & 7 & 46.7 \\
Autoempleados & & \\
Religión & 12 & 80 \\
Católica & 1 & 6.7 \\
Cristiana & 1 & 6.7 \\
Mormona & 1 & 6.7 \\
Testigos de Jehová & 1
\end{tabular}

Fuente: cuestionario de datos sociodemográficos

Se observa en la figura 1 de acuerdo a los resultados obtenidos en relación al factor de afrontamiento recursivo y centrado, el total $(100 \%)$ de la muestra manifestó alta capacidad para reunir la mayor información posible para solucionar el problema, algunas veces tratan de hacer que todo funcione a su favor, están atentos a cualquier cosa relacionada con la situación, y tratan de obtener más recursos para enfrentar el problema. Algunas veces tratan de ser creativos y proponer nuevas soluciones. Con tal de salir del problema algunas veces están dispuestos a cambiar su vida radicalmente. Lo que indica, que afrontan el problema, a través de información.

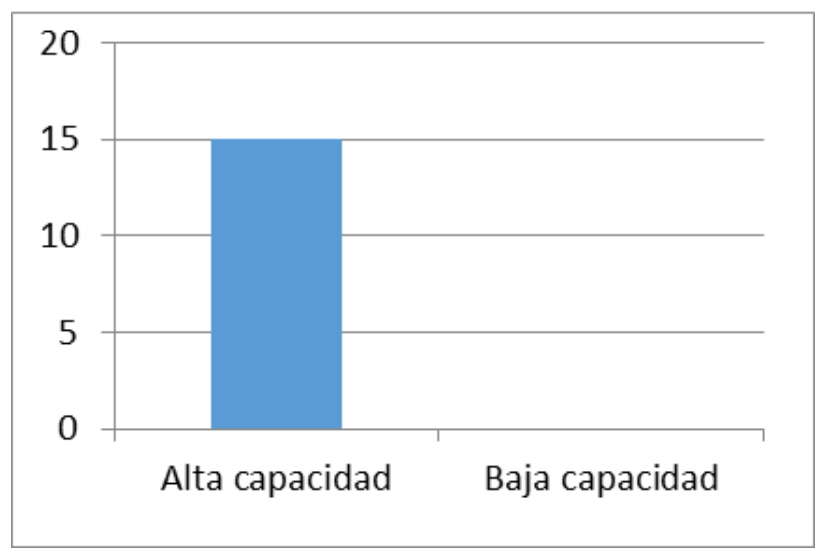

Figura 1 Factor recursivo y centrado 
Referente a la figura 2 con respecto al factor físico y enfocado: poco más de la mitad de la población (60\%) muestran un afrontamiento donde se observa baja capacidad para enfrentar un problema, dado que lo consideran demasiado complejo, refieren dificultad para completar tareas, reaccionan con exageración y solo pueden pensar en lo que les preocupa. Algunas veces tienden a culparse por cualquier dificultad que presentan y experimentan cambios en la actividad física. Sin embargo, el 40\% muestra alta capacidad para afrontar un problema del factor físico y enfocado.

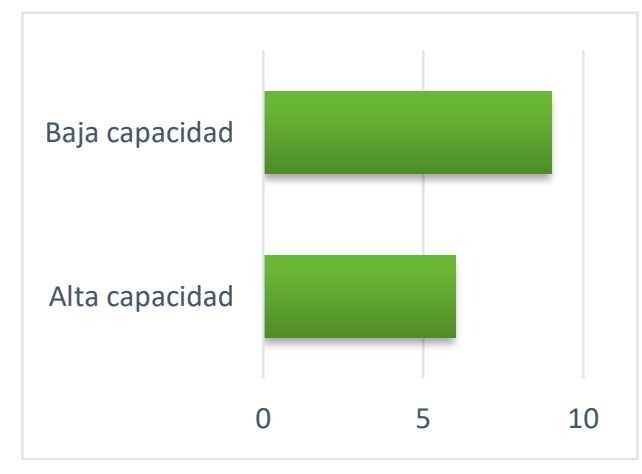

Figura 2 Factor físico y enfocado

Con respecto al factor de proceso de alerta, se observa en la figura 3 que casi el total (87\%) de los participantes refieren alta capacidad de alerta, en relación a que pueden manejar la situación analizando los detalles de tal como sucedió el problema. Algunas veces pueden seguir una gran cantidad de instrucciones a la vez y pueden adoptar rápidamente alternativas de solución, los participantes señalan que son menos efectivos bajo estrés,

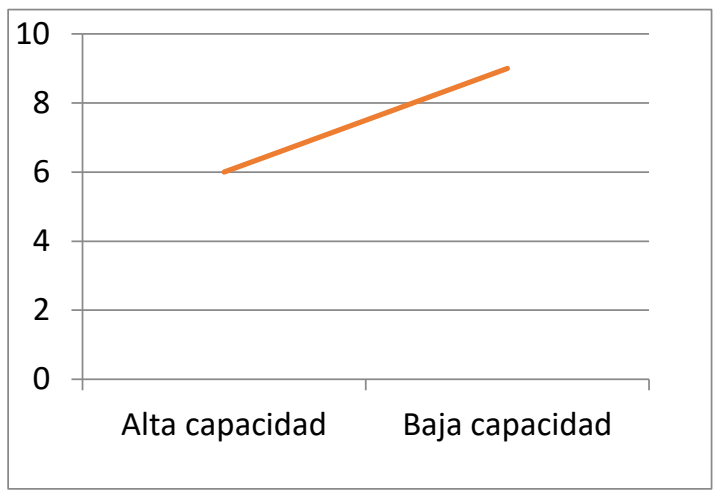

Figura 3 Factor de proceso de alerta
En relación a la figura 4 factor procesamiento sistemático los resultados arrojaron que más de la mitad de la muestra (60\%) presentan baja capacidad para identificar donde inicio el problema, algunas veces tratan de aclarar las dudas antes de actuar, piensan en el problema paso a paso y rara vez actúan hasta que tengan buena comprensión de la situación; mientras tanto el resto de la muestra (40\%) indica alta capacidad de llamar al problema por su nombre, analizan la situación y la miran como realmente es.

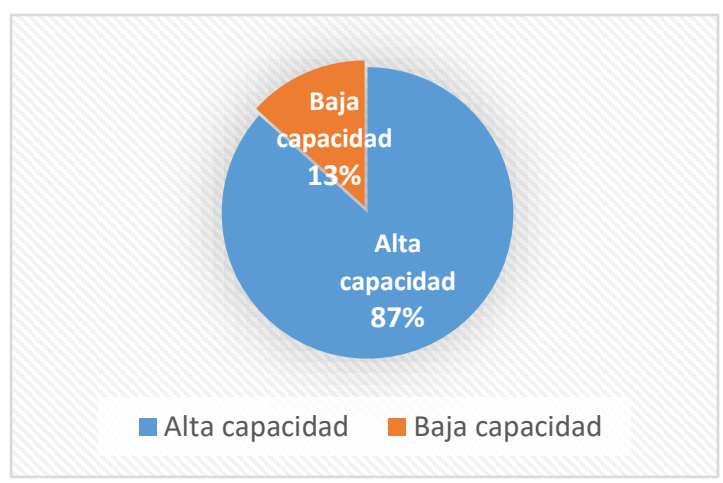

Figura 4 Factor Procesamiento sistemático

Se puede observar en la figura 5 el factor conociendo y relacionando donde casi una tercera parte $(73 \%)$ refieren alta capacidad en afrontar una actitud positiva ante las adversidades. Además, solucionan problemas en base a experiencias pasadas, recuerdan estrategias para solucionar problemas que en otras ocasiones les a ayudado y el $27 \%$ presenta baja capacidad para recordar las situaciones que ayudaron en problemas pasados.

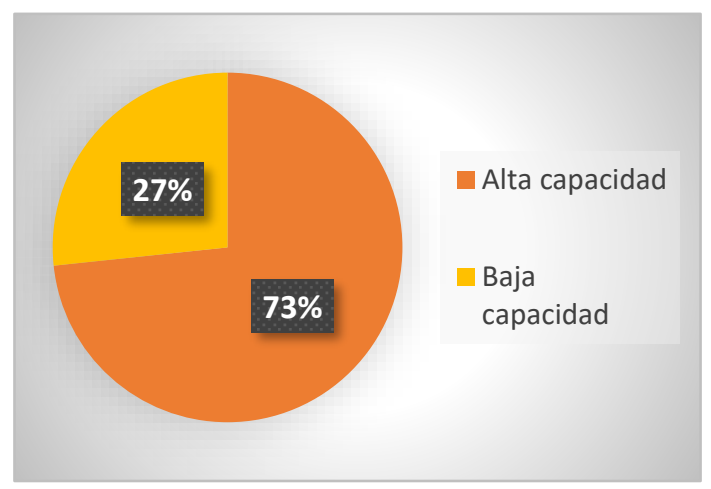

Figura 5 Factor Conociendo y relacionando 
Los datos generales arrojan que la capacidad de afrontamiento y adaptación en la mayoría de las personas con amputación (93\%) fue alta donde mostraron gran gama de estrategias para sobrellevar los problemas haciendo mayor hincapié en los factores recursivo y centrado con proceso de alerta. Así mismo una mínima parte $(7 \%)$ refleja baja capacidad de afrontamiento y adaptación en relación al factor físico y enfocado con procesamiento sistemático.

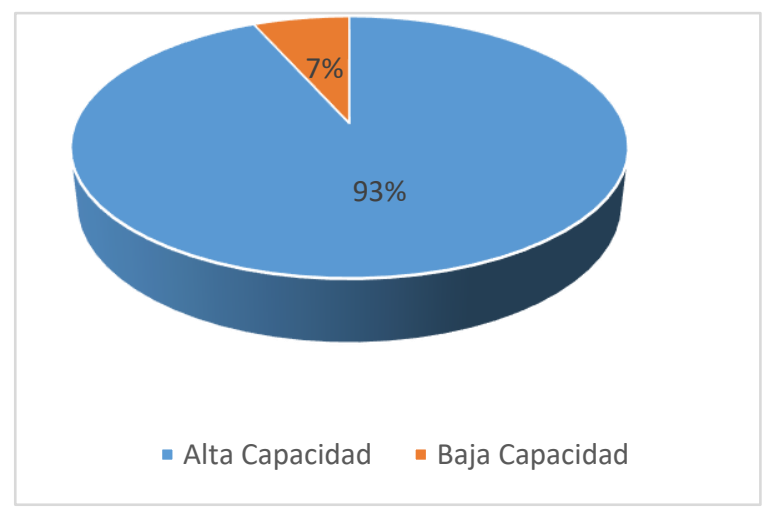

Figura 6 Capacidad de afrontamiento y adaptación en pacientes con amputación

\section{Discusión}

La adaptación es el medio por el cual las personas con pensamiento crítico individual o grupal, hacen uso del conocimiento y la parte consciente, para obtener la conformación humana y ambiental. Roy [5,6] menciona que el ser humano es altamente capaz de adaptarse a cualquier ambiente y crear cambios en él. En particular, para Roy se hace evidente en los pacientes de esta investigación, donde la mayoría de ellos presentan estrecha relación con su medio ambiente de manera favorable, en todas las situaciones que afrontan y se adaptan a la vida cotidiana.

Los participantes reflejan actitudes positivas para afrontar y solucionar problemas de forma creativa, reúnen la mayor cantidad de información posible para aumentar las opciones a fin de solucionar algún problema, atentos a cualquier cosa relacionada con la situación, hacen frente al problema desde un principio, basándose en las estrategias de solución utilizadas de experiencias pasadas, ensayan mentalmente una serie de acciones a seguir, analizan las consecuencias, aceptan las circunstancias estresantes y se empeñan en conseguir un cambio de una manera positiva; a lo que se encontró similitud de resultados con el estudio de Jiménez, [10] quien confirma que los usuarios de su investigación mostraron comportamientos de afrontamiento de alerta a cualquier cosa relacionada con la situación, actitud positiva de saber que estaban manejando el problema lo mejor que ellos podían; hacen frente a la situación desde un principio y se empeñan en buscar más de una solución a cualquier problema que enfrenten y su solución de respuesta se basa en experiencias de situaciones pasadas, lo que se observa que se afrontan y se adaptan al problema que presentan.

Situación contraria al estudio que realizo Trujillo [3] sobre el proceso de adaptación en adultos que sufrieron amputación y cuentan con una prótesis donde la muestra de estudio obtuvo baja capacidad de afrontamiento y adaptación; ya que frecuentemente presentan dificultad para completar tareas, encuentran el problema demasiado complejo y les es difícil identificar cual es el verdadero problema a tratar, así mismo piensan en el problema paso a paso y la mayoría de ellos se consideran con baja valía e incapaces de poder enfrentar la situación ante la amputación presentada,.

De acuerdo con Roy [5,6] cuando el usuario atraviesa por una situación estresante y es percibido como fuera de control, las personas llegan a utilizar estrategias de afrontamiento dirigidas a las emociones, lo cual hace más difícil encontrar una solución razonable; y como consecuencia a largo plazo poseen dificultades emocionales para adaptarse a su medio social, laboral y familiar, afectando de igual forma la adaptación de los familiares para lidiar con este proceso, lo cual fue contrario a este estudio donde los participantes encontraron estrategias de afrontamiento para brindar una solución al problema.

\section{Conclusión}

Los participantes entrevistados que presentaron amputación muestran una alta capacidad de afrontamiento y adaptación, sus principales tácticas van dirigidas a la solución de problemas, tratando de hacer que todo funcione a su favor, obteniendo más recursos para enfrentar la situación y haciendo frente a las situaciones desde el principio.

Entre los comportamientos más frecuentes se observó que obtuvieron alta capacidad de afrontamiento y adaptación fueron en el Factor Recursivo-centrado, proceso de alerta y el Factor Conociendo y Relacionando, donde sus comportamientos están focalizados en resolver y afrontar las diversas situaciones 
y problemas que se les presenten, mediante el aprendizaje de las soluciones que han funcionado para otros, mirando la situación positivamente como una oportunidad, utilizando el sentido del humor para manejar la situación.

De los factores que obtuvieron baja capacidad de adaptación y afrontamiento fue el factor: Físico y Enfocado y procesamiento sistemático encontrándose la dificultad de explicar cuál es el verdadero problema, facilidad para rendirse fácilmente, reaccionar con exageración al comienzo y solo pensar en lo que les preocupa.

Es importante la aplicación de teorías de mediano rango como la de adaptación de Callista Roy, en el que contribuye en el desarrollo del conocimiento de Enfermería y permite mejorar el cuidado holístico en este tipo de pacientes, basado en el conocimiento y comprensión, lo que ayuda a agilizar el proceso de afrontamiento y adaptación por el que cursan las diversas personas que han sufrido amputación.

\section{Limitaciones y Sugerencias}

El estudio tuvo ciertas limitaciones que son importantes considerar. Este estudio fue transversal, por lo tanto, ninguna afirmación acerca de causalidad puede ser hecha. La muestra fue pequeña, se sugiere que se aplique a poblaciones que estén sometidos a eventos estresantes. Solo se aplicó un instrumento. Por lo que se sugiere que se analicen variables fisiológicas para próximos estudios para relacionarlos.

\section{Referencias}

[1] Estadísticas del Dia Internacional de las personas con discapacidad.

INEGI [en línea] 2015 [citado octubre 2019] Recuperado en:

https://www.inegi.org.mx/contenidos/saladeprensa/aproposito/2015/discap acidad0.pdfhttps://www.inegi.org.mx/contenidos/saladeprensa/aproposito/ 2015/discapacidad0.pdf

[2] Vázquez E. Los amputados y su rehabilitación. Un reto para el Estado. [en línea] México, 2016 [citado 11 de septiembre de 2019] Recuperado

https://www.anmm.org.mx/publicaciones/ultimas publicaciones/Reha bilitacion.pdf
[3]Trujillo MA. Adriana Parrilla (dir) Proceso de adaptación en adulto que han sufrido una amputación y cuentan con una prótesis.

[Tesis en línea]. [Guatemala]: Universidad Rafael Landivar; 2012 [citado 22 de noviembre 2019] Recuperado a partir de: http://biblio3.url.edu.gt/Tesis/2012/05/42/Trujillo-Maria.pdf

[4] Cisneros N, Ascencio IJ, Libreros VN, Rodríguez H, Campos A, Dávila $\mathrm{J}$, et al. Índice de amputaciones de extremidades inferiores en pacientes con diabetes. [en línea] 2016 [citado 15 de agosto de 2019];54(4):472479. Recuperado en: https://www.medigraphic.com/pdfs/imss/im2016/im164k.pdf

[5] Roy, C., Andrews, A. The Roy adaptation model, 2nd ed., Stamford, CT, Appleton \& Lange, 1999. 574 p.

[6] Díaz L, Duran MM, Gallego P, Gómez B, Gómez E, González Y, Gutiérrez MC, Hernández A, Londoño JC, Moreno ME. Análisis de los conceptos del modelo de adaptación de Callista Roy. [en línea] 2002 [citado 16 de agosto de 2019]; 2(2):19-23. Recuperado en: https://aquichan.unisabana.edu.co/index.php/aquichan/article/view/18

[7] Nava DC, Olvera SS, Cadena JC. Atención de enfermería a un adulto con cardiopatía congénita basada en el modelo de adaptación de Roy. [en línea] 2015 [citado 29 de octubre de 2019];23(3):118-125. Recuperado en: https://www.medigraphic.com/pdfs/enfe/en2015/en153d.pdf

[8]. Alarcón MA. Modelo de adaptación de Roy aplicación de pacientes con diálisis peritoneal continua ambulatoria. . [en línea] 2007 [citado 15 de septiembre de 2019];15(3):155-160. Recuperado en: https://www.medigraphic.com/pdfs/enfermeriaimss/eim-

\section{7/eim073g.pdf}

[9] Lazcano M, Salazar BC. Adaptación en pacientes con diabetes mellitus tipo 2, según modelo de Roy. [en línea] 2009 [citado 15 de octubre de 2019];9(3):236-245. Recuperado en: https://aquichan.unisabana.edu.co/index.php/aquichan/article/view/15 $\underline{22 / 1968}$

[10] Jiménez VF, Zapata LS, Díaz L. Capacidad de afrontamiento y adaptación de los familiares del paciente adulto hospitalizado en una unidad de cuidados intensivos. [en línea] 2013 [citado 22 noviembre 
Publicación semestral, Educación y Salud Boletín Científico Instituto de Ciencias de la Salud Universidad Autónoma del Estado de Hidalgo, Vol. 9, No. 18 (2021) 133-140

de 2019];13(2):45-62. Recuperado en:

https://www.redalyc.org/pdf/741/74128688010.pdf

[11] Chumbes E, Meza N, Villegas R, Salazar Y (dir.) Factores de afrontamiento y adaptación del paciente que será sometido a cirugía plástica reconstructiva, en un Hospital de Lima. [tesis en línea]

[Lima]: Universidad Peruana; 2016 [citado 22 de noviembre 2019].

Recuperado a partir de:

http://repositorio.upch.edu.pe/bitstream/handle/upch/679/Factores\%2

0de\%20afrontamiento\%20y\%20adaptaci\%C3\%B3n\%20del\%20pacie

nte $\% 20 q u e \% 20$ ser $\%$ C3\%A1\%20sometido\%20a\%20cirug \%C3\%ADa

$\% 20 \mathrm{pl} \% \mathrm{C} 3 \% \mathrm{~A} 1$ stica\%20-

\%20reconstructiva\%2C\%20en\%20un\%20Hospital\%20de\%20Lima.p

df? sequence $=1 \&$ isAllowed $=\mathrm{y}$

[12] Gutiérrez C, Veloza M, Moreno M, Durán MM, López C, Crespo O. Validez y confiabilidad de la versión en español del instrumento Escala de medición del proceso de afrontamiento y adaptación de Callista Roy [en línea] 2007 [citado 15 de febrero de 2019];7(1):54-63. Recuperado en: http://www.scielo.org.co/pdf/aqui/v7n1/v7n1a05.pdf 